\title{
Employing Publically Available Biological Expert Knowledge from Protein-Protein Interaction Information
}

\author{
Kristine A. Pattin, Jiang Gui, and Jason H. Moore \\ Dartmouth Medical School, Lebanon, NH 03756, USA \\ kristine.a.pattin@dartmouth.edu \\ www.epistasis.org
}

\begin{abstract}
Genome wide association studies (GWAS) are now allowing researchers to probe the depths of common complex human diseases, yet few have identified single sequence variants that confer disease susceptibility. As hypothesized, this is due the fact that multiple interacting factors influence clinical endpoint. Given the number of single nucleotide polymorphisms (SNPs) combinations grows exponentially with the number of SNPs being analyzed, computational methods designed to detect these interactions in smaller datasets are thus not applicable. Providing statistical expert knowledge has exhibited an improvement in their performance, and we believe biological expert knowledge to be as capable. Since one of the strongest demonstrations of the functional relationship between genes is protein-protein interactions, we present a method that exploits this information in genetic analyses. This study provides a step towards utilizing expert knowledge derived from public biological sources to assist computational intelligence algorithms in the search for epistasis.
\end{abstract}

Keywords: GWAS, SNPs, Protien-protein interaction, Epistasis.

\section{Introduction: Challenges Confronting Genome-Wide Genetic Analysis}

The field of human genetics is advancing with the advent of new and cost efficient technology that allows us to rapidly generate large amounts of genomic data. It is now possible to measure a million or more SNPs at one time, however researchers are lacking methods to efficiently explore their results. The etiology of common human disease is understood to be complex, with multiple interacting genetic factors predisposing individuals to disease risk. To detect and characterize these epistatic, or gene-gene, interactions that confer disease susceptibility in such large scale studies requires the analysis of all pair-wise and higher-order combinations of SNPs. This poses a challenge that needs to be addressed before we are able to completely explore epistasis in GWAS in order to gain a more coherent understanding of the genetic architecture of a complex trait and its interacting elements [1]. 
The critical need for statistical and computational methods that are powerful enough to model the relationship between SNP interactions and disease susceptibility has been addressed by the development of numerous statistical, machine learning, and datamining techniques. Some programs of note are multifactor dimensionality reduction (MDR) [2], ReliefF [3] and random chemistry 4. Even though these methods have proven to be an effective way to model epistasis in smaller datasets, analysis of all higher-order SNP combinations in GWAS remains computationally infeasible.

One approach to this problem is to use computational intelligence algorithms that are able to explore a fitness landscape that is both vast and rugged. An important feature of this problem domain is that it is often the case that the attributes to be detected and modeled don't have detectable marginal effects and thus don't make good building blocks [5]. The use of statistical expert knowledge has been shown to improve the ability of learning algorithm to identify and exploit those building blocks that will yield an informative classifier Greene et al. (2008), have previously combined the power of ant colony optimization as a probabilistic learner with expert knowledge in the form of preprocessed ReliefF scores that reflect attribute quality and thus provide a measure of whether a particular attribute is a good building block [6] [7. Also Greene et al., (2009) and Moore et al., (2008) have shown that the same statistical expert knowledge incorporated in a computational evolution system (CES), has the same beneficial effect. They demonstrated that the system could learn to recognize and exploit a good source of expert knowledge from among several different options to discover optimal solutions in this problem domain [8],

cite2greene09. While preprocessed statistical knowledge is useful, it is likely not comprehensive in its ability to identify good building blocks. We anticipate that biological knowledge derived from biochemical pathways or regulatory networks of function, such as protein-protein interactions, will provide the complementary information that is needed to maximize the ability of a computational intelligence algorithm to identify optimal models of epistasis [10] 11]. The goal of the present study is to explore the bioinformatics methods that are necessary to extract and utilize expert knowledge from public protein-protein interaction databases.

For this study we develop metrics derived from PPI interactions found in the database, STRING (Search Tool for the Retrieval of Interacting Genes/Proteins). These are based on the confidence score for each interaction in the database [12] and used to to prioritize SNPs in a gene list that is derived from a real bladder cancer dataset. While reducing the size of a genomic dataset using this approach may be useful to conduct a more computationally efficient analysis, we wish to ultimately explore how this protein interaction information can be used to guide a computational intelligence algorithm.

\section{Materials and Methods}

\subsection{STRING}

This study aims to understand how we can utilize expert knowledge from proteinproteins interactions to guide the search for epistasis in GWAS. We extract our 
biological expert knowledge from STRING (Search Tool for the Retrieval of Interacting Genes/Proteins). The newest version of STRING, 8.2, represents over 2.5 million proteins from 630 different organisms, and incorporates PPI information from a number of interaction databases such as the Human Protein Reference Database (HPRD), BioGrid, the Molecular Interaction Database (MINT), the Biomolecular Interaction Network Database (BIND) which is a component of the Biomolecular Object Network Database(BOND), the Database of Interacting Proteins (DIP), and also imports known reactions from Reactome and Kyoto Encyclopedia of Genes and Genomes (KEGG) pathways. Recent additions to this database incorporate information from IntACT, EcoCyc, NCI-Nature Pathway Interaction Database and the Gene Ontology (GO). Automated text-mining of PubMed abstracts, Online Mendelian Inheritance in Man (OMIM), and data from other databases such as the Saccharomyces Genome Database, Wormbase, and the Interactive Fly supplement this information [13]. At the time of our study however, we utilized the then current version available for download, version 7.0. Each interaction has a combined confidence score that ranges from 0 to 1 and are based on each source of evidence. Computing the combined confidence score, which we use as our source of expert knowledge, is a simple expression of the individual scores for each source of evidence. For a detailed description of the scoring methods see (von Mering et al., 2005) [12].

\subsection{Interaction Scenario Simulation}

In order to develop and test our method, we identified 8 different gene-gene interaction scenarios present in a list of genes derived from a real genetic bladder cancer dataset. The purpose of this was to represent a range of different genegene interaction scenarios that had validated interactions in STRING so that we may determine how PPI interaction information can be used as a source of expert knowledge should these biological interactions represent actual statistical epistatic interactions. Therefore, these interactions represent only theoretical statistical interactions. The original genetic data were originally collected for the purpose of assessing genetic risk factors of bladder cancer (Andrew et al., 2008). This dataset genotyped 491 cases and 791 controls across 1,423 SNPs found in 394 genes [14.

The gene-gene interactions designated in each scenario were selected to represent a range of combined confidence scores. As described, von Mering et al.,(2005) have developed the scoring system for PPIs in STRING and consider a score $<0.7$ and $>0.4$ as a medium confidence interaction 12 . The default threshold for querying interactions in STRING is 0.4 , and we kept this setting when submitting the entire list of genes in our bladder cancer dataset to STRING. We chose interactions that exhibited a range of confidence for each interaction: BARD1BRCA1 (confidence $=0.999)$, CASP3-CASP9 $($ confidence $=0.999)$, IL4R-IL6R (confidence $=0.825)$, RET-ENG (confidence $=0.7)$, TERT-MTR (confidence $=0.532$ ), HSD3B1-SOD1 (confidence $=0.497$ ), and two pairs that showed no existence of interaction in STRING, DRD4-BIC and RERG-SCUBE2 [Table 1 . 
Table 1. PPI interaction pairs represented in our bladder cancer data. The combined confidence score from STRING for each interaction and the number of interactors for each gene. Each of the interaction pairs was chosen to represent a range of interaction scores and diverse interaction scenarios.

\begin{tabular}{|c|c|c|}
\hline PPI & Confidence Score & \# of Interactors $\geq 0.4$ \\
\hline CASP9 & 0.999 & 29 \\
CASP3 & & 58 \\
\hline BARD1 & 0.999 & 17 \\
BRCA1 & & 71 \\
\hline IL6R & 0.825 & 24 \\
IL4R & & 38 \\
\hline ENG & 0.7 & 12 \\
RET & & 13 \\
\hline MTR & 0.532 & 7 \\
TERT & & 18 \\
\hline SOD1 & 0.497 & 15 \\
HSD3B1 & & 8 \\
\hline BIC & None & 0 \\
DRD4 & & 0 \\
\hline SCUBE2 & None & 0 \\
RERG & & 0 \\
\hline
\end{tabular}

Note that if any disease or pathway relationship exists between these genes it is by chance, given that genes were not selected on this basis.

\subsection{Metrics}

Expert knowledge from protein-protein interactions was employed to develop metrics that we used prioritize genes in a genomic dataset. The goal was to determine if PPIs will provide a valuable source of expert knowledge to preprocess data and eventually evaluate the effectiveness of this approach in a computational intelligence algorithm. The combined confidence score for protein-protein interactions from the STRING database was used to prioritize the genes a bladder cancer gene list according to each metric we developed. Note that all SNPs represented in the bladder cancer dataset that are found in the same gene are assigned the same metric score and rank using this method.

First, all 394 genes in the bladder cancer dataset were queried to obtain the list of combined confidence scores for all existing interactions between genes that represent PPIs in STRING. Using this combined confidence score, metrics were developed as follows:

For gene $\mathrm{X}$ that interacts with $\mathrm{N}$ number of genes with descending confidence scores $n_{1} \ldots n_{i}$ we computed,

Average(AVE),

$$
\operatorname{AVE} X=\frac{\sum\left(n_{1} \ldots n_{i}\right)}{N}
$$


the average of all confidence scores for a gene and its interaction partners, and

$$
\begin{gathered}
\operatorname{Sum}(\mathrm{SUM}), \\
\operatorname{SUM} X=\sum\left(n_{1} \ldots n_{i}\right),
\end{gathered}
$$

the sum of all confidence scores for a gene and its interaction partners.

While the maximum confidence score was evaluated as a metric, it was discovered that a considerable number of genes are represented by the same maximum confidence score. This was not useful for the individual prioritization of all genes, thus two additional metrics, MAX-SUM and MAX-AVE were developed. In this case, the metric score for each gene is represented by the numerical rank for that gene in the dataset after prioritization. These were developed as follows:

For gene $X_{1} \ldots X_{i}$, those with the same maximum confidence score are sorted in descending order by their SUM $X$ or by AVE $X$.

The MAX-SUM metric becomes the numerical rank of the gene as they are prioritized in descending order.

To determine if using a specific cut-off of 0.4 had any effect on the metric scores for each gene, we recalculated each metric using lower thresholds of 0.2 and 0.3 , and higher thresholds of $0.5,0.6,0.7$, and 0.8 . Metrics calculated using these thresholds were compared with each other and also to our original metrics. The comparison was performed using the Wilcoxon rank sum test as described in the next section, however, significant difference was not observed after implementing these threshold cutoffs (data not shown).

\subsection{Evaluating Metrics}

To evaluate these metrics, genes in the bladder cancer dataset were prioritized by their metric score and evaluated by extent by which each metric could reduce the entire gene list while retaining each of the 8 validated interaction pairs. This included two scenarios where genes had no evidence of interacting in STRING. Genes were sorted in descending order after being assigned their individual metric scores. The numerical position of each gene within the prioritized list represents the rank for that gene and is expressed in terms of the number of SNPs included in the gene. Since the actual bladder cancer dataset is comprised of SNP data, all SNPs in one gene share this rank. This means that genes with a higher metric score would have a smaller numerical rank. For example, the highest scoring gene in the list would be 1 , but may have 5 SNPs within that gene, therefore making the rank for this gene, 5 .

The gene list was then truncated at the lowest scoring gene in each interaction pair so that both of these genes, inclusive of all their SNPs, were maintained in the truncated list. For example, expressing the size of this list in terms of SNPs, applying the AVE metric to the CASP3 - CASP9 scenario, if we truncated the list at CASP3, this would include 315 SNPs, (315 being considered its rank) but this would omit its interaction partner CASP9 since it had a larger numerical rank. To include CASP9, the list had to be truncated at 599 SNPs. This larger 
numerical rank for each interaction pair was the number we used in order to compare the metrics, not the metric score itself.

The ability of each metric to significantly reduce the gene list in each interaction scenario was evaluated using a pair-wise one sided Wilcoxon rank sum test to compare the ranks obtained by each metric.A p-value of $<0.05$ was considered to be indicate significant reduction. The non-interacting SNPs were not included in this comparison, because it was confirmed that for all metrics, their rank was 0, and for both pairs to be included in the gene list, all 1423 SNPs had to be considered. This was expected since these scenarios served as control to assure that our computation and ranking system were functioning appropriately.

We also examine whether there was a correlation between the confidence score for a given interaction and the ranking for the pairs across all metrics. To evaluate this we performed a Spearman Rank correlation test and considered there to be a significant correlation for p-values $<0.05$.

\subsection{Bladder Cancer Data}

Finally, metrics were examined in the context of the actual gene-gene interactions that were indentified in a variation of the bladder cancer dataset utilized throughout this study. Andrew et al. (2008) used a multifactor analysis strategy to investigate associations between DNA repair polymorphisms and bladder cancer risk. Gene-environment and gene-gene interactions were evaluated using logistic regression, MDR, hierarchical interaction graphs, classification and regression trees, and logic regression analyses. All methods supported an interaction between DNA repair polymorphisms XRCC1-399/XRCC3-241 ( $\mathrm{p}=0.001)$, and three methods identified an interaction between XRCC1-399/XPD-751 (p $=0.008)$. The ranks of these gene pairs using each of our metrics were calculated to determine if they would have been effective in this real genetic analysis.

\section{Results}

In total there were 357 interacting proteins within the dataset and a total of 3,921 different interactions amongst these proteins. A full list of all interactions and their individual confidence score and the metric score for each individual gene are available upon request.

Figure 11demonstrates the extent to which each metric was able to reduce the size of the gene list for each of the 6 interaction scenarios. The confidence score for each gene pair interaction is represented on the $\mathrm{x}$-axis in ascending order, and the ranking for each of these is represented on the y-axis. This ranking represents the extent to which the gene list could be reduced by applying each metric. Table 2 displays the individual rankings produced for each scenario after being prioritized by each metric. In a vast majority of the cases, higher confidence interactions are found in smaller subsets of the gene list when all metrics are applied, however this is not a consistent trend. For example, even though HSD3B1-SOD1 has the lowest confidence score, it is found in a smaller subset of the list than RET-ENG (confidence=0.7) when using AVE, MAX-SUM, and MAX-AVE. Both SUM and 
AVE produce larger subsets of the gene list, except for in the case of the RETENG scenario for both and also in the case of CASP9-CASP3 for SUM. The MAX-SUM metric produces similar ranks as MAX-AVE, yet these decrease as confidence score increases.

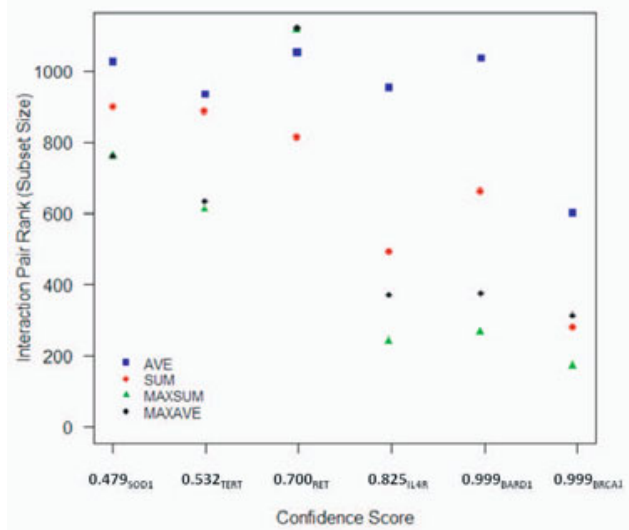

Fig. 1. Depicts the extent to which each metric was able to reduce the gene list for each of the 6 functional interactions. We did not include the non interacting pairs. The confidence score for each SNP pair interaction is represented on the $\mathrm{x}$-axis in ascending order, and the ranking for each interacting SNP pair is represented on the y-axis. The rank is also indicative of the subset size to which the gene list was reduced.

To determine if these observations were significant, a one sided pair-wise Wilcoxon rank sum test was used to compare each metric in terms of the gene rankings they produced across the interaction scenarios [Table(3)]. The metric AVE produced significantly larger ranks than SUM, MAX-SUM, and MAX$\operatorname{AVE}(\mathrm{p}=0.017,0.031,0.031)$. This means that compared to these 3 metrics, AVE was not able to reduce the gene list as effectively as the others while retaining the interaction pairs. SUM showed no significant difference from MAXSUM, or MAX-AVE, yet as mentioned, produced smaller rankings than AVE. MAX-SUM, however produced the lowest mean rank amongst the metrics (525) and also had significantly lower ranks than AVE and MAX-AVE ( $p=0.031$ and $\mathrm{p}=0.030$ ). When examining the individual numerical pair ranks produced by SUM and MAX-SUM, it is clear that all the ranks for MAX-SUM are much smaller than SUM except in the case of the rank of RET-ENG. We determined this to be the cause of the lack of significant difference between the two metrics. Note that SUM showed no significant difference compared to MAX-AVE either on account of the smaller rank score it produced for RET-ENG. Overall, MAX-SUM was able reduce the size of the gene list to the greatest extent in a majority of the interaction scenarios as compared to the other metrics despite the lack of significant difference between this metric and SUM. 
Table 2. Shows the ranking for each interaction pair which is also the subset size of the gene list after genes were prioritized by each metric. The non-interacting SNPs do not have any interactors so therefore will only be included in the gene list as a whole since they cannot be ranked by the metrics.

\begin{tabular}{|c|c|c|c|c|}
\hline PPIs & AVE & SUM & MAX-AVE & MAX-SUM \\
\hline CASP3.CASP9 & 599 & 279 & 313 & 169 \\
\hline BARD1.BRCA1 & 1032 & 661 & 374 & 264 \\
\hline IL4R.IL6R & 950 & 491 & 370 & 239 \\
\hline RET.ENG & 1048 & 811 & 1118 & 1112 \\
\hline TERT.MTR & 931 & 885 & 632 & 608 \\
\hline HSD3B1.SOD1 & 1022 & 898 & 758 & 758 \\
\hline DRD4.BIC & 1423 & 1423 & 1423 & 1423 \\
\hline RERG.SCUBE2 & 1423 & 1423 & 1423 & 1423 \\
\hline
\end{tabular}

Examining the relationship between the confidence of an interaction and its rank, it's observed that while an interaction with a higher confidence score may have a lower rank, this was not the case for all scenarios. However, there was a significant relationship between the two by means of Spearman Rank Correlation test. This showed that there was a negative correlation between the numerical rankings produced by each metric $(\mathrm{p}<0.05)$, except AVE. Rank increased as the confidence score of the interacting pairs decreased [[Table(3)].

Table 3. Shows the metrics as compared to each other by one-sided pair-wise Wilcoxon rank sum test. Significance is indicated by $(*)$ and directionality listed below.

*AVE > SUM, MAXSUM, MAXAVE

*MAXSUM $<$ AVE, MAXAVE

$*$ MAXAVE $<$ AVE

\begin{tabular}{|c|c|c|c|c|c|c|}
\hline$\cdot$ & AVE & SUM & MAX-SUM & MAX-AVE & Spear. P-Val. & Corr. Coeff. \\
\hline AVE & $\cdot$ & $\cdot$ & $\cdot$ & $\cdot$ & 0.40 & -0.15 \\
\hline SUM & $0.016^{*}$ & $\cdot$ & $\cdot$ & $\cdot$ & $0.007^{*}$ & -0.9 \\
\hline MAX-SUM & $0.031^{*}$ & 0.160 & $\cdot$ &. & $0.05^{*}$ & -0.72 \\
\hline MAX-AVE & $0.031^{*}$ & 0.290 & $0.030^{*}$ & $\cdot$ & $0.05^{*}$ & -0.72 \\
\hline
\end{tabular}

We applied our metrics to the bladder cancer dataset in the context of the interactions identified by Andrew et al. (2008). These results are described in [Table (4)]. Both interactions, XRCC1-XPD and XRCC1-XRCC3, were supported in STRING with confidence scores of 0.930 and 0.774 , respectively. We find that by applying the SUM and AVE metrics in both cases, the dataset could be reduced to between 288 and 346 SNPs. However unlike in the simulated interaction scenarios, MAX-AVE and MAX-SUM produced larger ranks for these pairs and would have only been able to reduce the dataset to between 1126 and 1158 SNPs from the original 1423 SNPs represented. 
Table 4. Shows the pair ranking for the gene-gene interactions identified by Andrew et al. (2008) when each metric was applied to their bladder cancer data. The interactions identified were between SNPs in each of the DNA repair genes listed.

\begin{tabular}{|c|l|l|c|c|}
\hline DNA Repair Gene Interactions & AVE & SUM & MAX-AVE & MAX-SUM \\
\hline XRCC1.XRCC3 & 288 & 346 & 1126 & 1176 \\
\hline XRCC1.XPD & 288 & 234 & 1130 & 1158 \\
\hline
\end{tabular}

\section{Discussion}

The goal of our study was to develop metrics based on protein-protein interaction information that would allow us to prioritize the SNPs in genomic datasets. We consider this a first step towards understanding how we can utilize expert knowledge derived from public biological sources, such as PPI databases, to facilitate the search for epistasis in GWAS. We use the combined confidence score for PPIs in STRING to develop these metrics and applied them to a real bladder cancer dataset from which we derived different gene-gene interaction scenarios. We found that of the four metrics that we have developed (AVE, SUM, MAX-SUM, and MAX-AVE), MAX-SUM was able to reduce the gene list in our datasets to the greatest extent across a majority of the interaction scenarios. While we did see a negative correlation between ranks and confidence score, except when AVE was used, [Table(3)] with higher confidence interactions typically being found in smaller subsets of the gene list, we recognize that the nature of the interaction may greatly influence this.

This was further supported by what we observed when we applied our metrics to the real interaction scenarios identified by Andrew et al. (2008). In our simulated scenarios, SUM and AVE produced larger subset sizes than MAX-SUM and MAX-AVE. However, it appears as though SUM and AVE would have been ideal to apply to the bladder cancer dataset in terms of the real statistical interactions that were observed. These metrics were able to reduce the dataset to a size that was approximately four times smaller in both cases, and despite the different confidence level of the interactions, 0.774 and 0.993 . While this demonstrates that one metric may not be particularly more useful over the others, we show that expert knowledge from PPIs could have been applied to this study to narrow the scope of the analysis and still obtain the results that were previously published.

The diversity of the interaction scenarios [Table (1)] is why we do not see one particular metric consistently reducing the gene list, and also why interaction scenarios of higher confidence are not always included in the smaller subsets of the gene list, such as in the case of RET-ENG.

For the RET-ENG scenario, we see that the pair is included in the largest subsets produced by all metrics except SUM, even though it has a confidence score of 0.7 . We find that RET interacts with 13 proteins and ENG with 12 . However, out of these interactions, there are few high confidence interactions, especially for RET. More than half of its interactions have a confidence score under 0.7. Also, RET has a lower maximum confidence score of 0.874 which automatically ranks it as 1118 
on the entire gene list inclusive of all SNPs. As mentioned, since the pair ranks are based on the gene with the higher numerical rank in the pair, we see this pair included in larger subsets due to the lack of a higher maximum score for RET using MAX-SUM and MAX-AVE. While the AVE score for its interactions might also be fairly low, it does have a considerable number of interaction partners, allowing for it to achieve a high metric score using SUM.

These examples and our application to the real statistical interactions exhibit why we chose not to consider one metric as the "best" metric, and acknowledge that there is room for exploration concerning how the combined protein interaction score from STRING can be utilized as biological expert knowledge. All metrics have the potential provide useful information to genetic studies, and we have shown that two of our metrics would have been able to effectively reduce the bladder cancer dataset while retaining the epistatic interactions that were previously shown to confer disease risk.

\section{Conclusion}

To exhaustively search thousands to millions of SNP combinations would not be practical due to the computational intensity involved in the process, and our work demonstrates one method that can be used to facilitate this search. The use of expert knowledge for these purposes is not a novel idea and has proven successful in similar endeavors [15] 16] 17] 18. However, there are potential drawbacks to using information from PPIs that should be acknowledged.

Certainly, there are inherent biases in any protein interaction database. This is something that is unavoidable and partially results from the ability of different experimental methods to capture different types of interactions. Also, a handful of proteins may be more widely studied. These facts influence the amount of evidence, whether it be via literature or other sources, that in turn determine the confidence placed in an interaction. Also, some may argue that by using expert knowledge, we are biasing a study, instilling the need for a prior hypothesis. While this may be valid, regardless we still do not have the appropriate computational power to explore higher order epistatic interactions in genome-wide datasets. By acknowledging the potential database biases, a researcher has the power to take these biases into consideration when conducting their analysis and understand how, if at all, this may have influenced their results.

We have shown that the protein interaction confidence score in the STRING database can be represented in a number of ways that may indicate the validity of an interaction as well as how central a gene is to the dataset based on the number of interactors it has within that dataset. If this holds true and there is biological representation of statistical results in an analysis, the application of our methods for prioritizing SNPs and reducing the scope of the analysis has a higher probability of retaining functional epistatic interactions after the data is processed. The development of computational methods that aid in the discovery and characterization of epistatic interactions in GWAS is of great importance, and this study opens the door for the utilization of expert knowledge from PPIs to guide these methods. 
While these metrics are useful for preprocessing GWAS data, it is possible we may be removing potentially important information by truncating a dataset. To this end, we additionally would like to explore how to use this form of biological expert knowledge in the computational evolution system we have developed (CES), that has the ability to identify complex disease-causing genetic architectures in simulated data by exploiting valid sources of statistical expert knowledge. Unlike preprocessed data, all SNPs in the dataset would be considered in this case. We anticipate that this biological expert knowledge will improve the performance of this system.

Ultimately, we hope that the use of this biological expert knowledge will provide a complement to the statistical expert knowledge that we have already shown to be successful in such a system. This can supplement an analysis with a foundation for interpreting epistatic models and understanding how they influence disease risk biologically.

\section{References}

1. Moore, J., Ritchie, M.: The challenges of whole-genome approaches to common diseases. JAMA 29, 1642-1643 (2004)

2. Ritchie, M., Hahn, L., Roodi, N., et al.: Multifactor dimensionality reduction reveals high-order interactions among estrogen metabolism genes in sporadic breast cancer. Am. J. Hum. Genet. 69, 138-147 (2001)

3. Moore, J., White, B.: Tuning reliefF for genome-wide genetic analysis. In: Marchiori, E., Moore, J.H., Rajapakse, J.C. (eds.) EvoBIO 2007. LNCS, vol. 4447, pp. 166-175. Springer, Heidelberg (2007)

4. Eppstein, M., Payne, J., White, B., Moore, J.: Genomic mining for complex disease traits with 'random chemistry'. Genetic Programming and Evolvable Machines 8, 395-411 (2007)

5. White, B., Gilbert, J., Reif, D., et al.: A statistical comparison of grammatical evolution strategies in the domain of human genetics. In: Proceedings of the IEEE Congress on Evolutionary Computing, pp. 676-682 (2005)

6. Greene, C., White, B., Moore, J.: Ant colony optimization for genome-wide genetic analysis. In: Dorigo, M., Birattari, M., Blum, C., Clerc, M., Stützle, T., Winfield, A.F.T. (eds.) ANTS 2008. LNCS, vol. 5217, pp. 37-47. Springer, Heidelberg (2008)

7. Greene, C., Gilmore, J., Kiralis, J., et al.: Optimal use of expert knowledge in ant colony optimization for the analysis of epistasis in human disease. In: Pizzuti, C., Ritchie, M.D., Giacobini, M. (eds.) EvoBIO 2009. LNCS, vol. 5483, pp. 92-103. Springer, Heidelberg (2009)

8. Moore, J., Andrews, P., Barney, N., et al.: Development and evaluation of an openended computational evolution system for the genetic analysis of susceptibility to common human diseases. In: Marchiori, E., Moore, J.H. (eds.) EvoBIO 2008. LNCS, vol. 4973, pp. 129-140. Springer, Heidelberg (2008)

9. Greene, C., Hill, D., Moore, J.: Environmental sensing of expert knowledge in a computational evolution system for complex problem solving in human genetics. In: Riolo, R., O-Reilly, U.-M., McConaghy, T. (eds.) Genetic Programming Theory and Practice, vol. VII, pp. 19-36. Springer, Heidelberg (2009)

10. Pattin, K., Moore, J.: Exploiting the proteome to improve the genome-wide genetic analysis of epistasis in common human diseases. Hum. Genet. 124, 297-312 (2009) 
11. Pattin, K., Moore, J.: Role for protein-protein interaction databases in human genetics. Exp. Rev. Proteomics 6, 647-659 (2009)

12. von Mering, C., Jensen, L., Snel, B., et al.: STRING: known and predicted proteinprotein associations, integrated and transferred across organisms. Nucleic Acids Res. 1(33), D433-D437 (2005)

13. Jensen, L., Kuhn, M., Stark, M., et al.: STRING 8-a global view on proteins and their functional interactions in 630 organisms. Nucleic Acids Res. 37, D412-D416 (2009)

14. Andrew, A., Karagas, M., Nelson, H., et al.: Assessment of multiple DNA repair gene polymorphisms and bladder cancer susceptibility in a joint Italian and U.S. population: a comparison of alternative analytic approaches. Hum. Hered. 65, 105-118 (2008)

15. Emily, M., Mailund, T., Hain, J., et al.: Using biological networks to search for interacting loci in genome-wide association studies. Eur. J. Hum. Genet. 17(10), 1231-1240 (2009)

16. Bush, W., Dudek, S., Ritchie, M.: Biofilter: a knowledge-integration system for the multi-locus analysis of genome-wide association studies. In: Pac. Symp. Biocomput., pp. 368-379 (2009)

17. Shriner, D., Tesfaye, B., Padilla, M., et al.: Commonality of functional annotation: a method for prioritization of candidate genes from genome-wide linkage studies. Nucleic Acids Res. 36(4), e26 (2008)

18. Saccone, S., Saccone, N., Swan, G., et al.: Systematic biological prioritization after a genome-wide association study: an application to nicotine dependence. Bioinformatics 24, 1805-1811 (2008) 